\title{
Verfassungsrechtspluralismus in West Sumatra: Veränderungen in staatlicher und dörflicher Verfassung im Zuge der Dezentralisierung in Indonesien ${ }^{1}$
}

\author{
Von Franz, von Benda-Beckmann, Halle/Saale
}

\section{Einleitung}

Die Beziehungen zwischen staatlichem Verfassungsrecht und lokalen verfassungsrechtlichen Ideen haben in fast allen Ländern in der Dritten Welt eine lange Geschichte. Diese begann spätestens, als die dortigen politischen Organisationen in die neuen kolonialen Staatsverbände eingebunden wurden. Elemente lokaler Herrschafts- und Verfassungsformen wurden zwar im Rahmen der indirekten Herrschaftspolitik der meisten Kolonialstaaten teilweise anerkannt, allerdings unter dem Primat der staatlichen Souveränität, welche nach staatlich rechtlichem Denken und Handeln die politische Dimension der lokalen politischen Organisationen absorbierte. Damit wurden diese traditionellen Herrschaftsstrukturen in vielen Staaten sehr geschwächt, in jedem Fall verändert; sie sind jedoch nur selten ganz verschwunden. Die in den letzten Jahren in vielen Staaten der Dritten Welt angestrebte Dezentralisierung hat die hiermit zusammenhängenden Probleme wieder deutlich in den Vordergrund gerückt. ${ }^{2}$

Leicht überarbeitete Fassung der Herbert-Krüger-Gedächtnisvorlesung auf der 27. Jahrestagung des Arbeitskreises für Überseeische Verfassungsvergleichung vom 28.-30. Juni 2002 in Halle/Saale. Er beruht überwiegend auf der gemeinsamen Forschung mit K. von Benda-Beckmann in West Sumatra und den bisher erarbeiteten Publikationen und Konferenzbeiträgen: $F$. und $K$. von Benda-Beckmann, Recreating the nagari: Decentralisation in West Sumatra, Halle, 2001; dies., Actualising history for binding the future: Decentralisation in Minangkabau, in: P. Hebinck / G. Verschoor (eds.), Resonances and dissonances in development: Actors, networks and cultural repertoires, Assen, 2001, 33-47.

Für Afrika siehe unter anderem E.A.B. Rouveroy van Nieuwaal / D.I. Ray (eds.), The new relevance of traditional authorities to Africa's future, Special Issue of the Journal of Legal Pluralism, Vol. 37-38 (1996); E.A.B. Rouveroy van Nieuwaal / W. Zips (eds.), Sovereignty, legitimacy, and power in West African societies: Perspectives from legal anthropology, Hamburg, 1998. 
Dies ist auch in Indonesien der Fall. Seit dem Ende des Suharto-Regimes im Jahre 1998 und dem Beginn der sogenannten Orde Reformasi verfolgt Indonesien eine Dezentralisierungspolitik. Die Strukturen dieser Politik sind in zwei 1999 erlassenen Gesetzen - über regionale Autonomie und über die finanziellen Beziehungen zwischen Zentrum und den Regionen - niedergelegt. Das neue Autonomiegesetz kennt drei autonome Verwaltungsebenen: den Zentralstaat, die Distrikte (Kabupaten) und die Dörfer. Nach dem neuen Gesetz wird die Zentralverwaltung reduziert. Nur die Bereiche der Außenpolitik, der Finanzpolitik, der Justiz, Religion und der Verteidigung bleiben zentrale Ministerien. Zusätzlich bleiben bestimmte Dienste auf zentraler Ebene tätig, wie die Regelung der staatlichen Verwaltung, der makroökonomischen Planung, der Technologiepolitik und des Umwelt- und Naturschutzes. Die anderen zentralen Ministerien werden abgeschafft, ihre Befugnisse und Aufgaben überwiegend den Distrikten übertragen. Die Provinzen, bis zur Verabschiedung der neuen Gesetze die zweitmächtigste Verwaltungsebene, haben keine eigenständige Autorität mehr und sind zur Außenstelle des Zentrums und zu Koordinatoren der Distrikte degradiert. Die früheren Sub-Distrikte (Kecamatan) sind als eigenständige Verwaltungseinheiten aufgehoben und direkt der Distriktsverwaltung unterstellt. ${ }^{3}$

Diese Politik ist das Ergebnis von regionalen und globalen politischen Prozessen. Sie entspringt teils lokalen politischen und wirtschaftlichen Aspirationen, vor allem der reicheren Regionen Indonesiens, die in einigen extremen Fällen mit Bestrebungen nach weitgehender Autonomie oder selbst Unabhängigkeit einhergehen. Vor allem die Regionen außerhalb Javas sind nicht länger zu den großen Abgaben an den Zentralstaat und der großen Umverteilung zwischen den Regionen bereit, wie sie während des Suharto-Regimes üblich waren. Seitens des Zentrums war die Wahl der Distrikte, und nicht der Provinzen, als wichtige neue autonome Einheit vor allem durch die Furcht eingegeben, dass sonst sezessionistische Bestrebungen unterstützt würden. Die Dezentralisierung ist jedoch auch weitgehend unter dem Druck internationaler Organisationen wie der Weltbank und des Internationalen Währungsfonds zu Stande gekommen. Diese und andere ausländische Geber, unter anderen die Gesellschaft für Technische Zusammenarbeit (GTZ), unterstützen die Dezentralisierung mit finanzieller und technischer Hilfe. Dezentralisierung, gerade in

Für weitere Informationen über die Dezentralisierung in Indonesien siehe Asia Research Centre, Decentralisation and development cooperation: Issues for donors, Asian Research centre, Murdoch University for The Australian Agency for International Development (AUSAID) of the Department of Foreign Affairs and Trade, 2001; D.F. Anwar, The fall of Suharto - Understanding the politics of the global. Paper presented at the 3rd EUROSEAS Conference in London, 6-8 September 2001; H. Antlöv, Village governance and local politics in Indonesia. Paper presented at the 3rd EUROSEAS Conference in London, 6-8 September 2001; A. Booth, Indonesia: Will decentralisation lead to disintegration?, Paper presented at the 3rd EUROSEAS Conference in London, 6-8 September 2001; S. Usman, Indonesia's decentralisation policy: Initial experiences and merging problems. Paper presented at the 3rd EUROSEAS Conference in London, 6-8 September 2001. Siehe auch die sehr informative website der GTZ, http://www.gtzfdm.or.id. 
einem bislang sehr zentralistisch und autoritär regierten Staat, verspricht ein Mehr an Demokratie und Eigenverantwortung regionaler und lokaler Verwaltungseinheiten. ${ }^{4}$

Die Situation in der Provinz West-Sumatra ist besonders interessant, weil die Dezentralisierungspolitik hier aufgenommen wurde, um auch zu einer weitgehenden Reform der untersten, dörflichen Verwaltungsstrukturen zu kommen. Bis 1983 baute die unterste Verwaltungsstruktur auf den traditionellen politischen Einheiten der Minangkabauschen Gesellschaft auf, den Nagari, oft Dorfrepubliken genannt. 1983 wurde das Modell der Desa-Dörfer aus Java importiert, was dazu führte, dass alle Nagari in mehrere Desa aufgeteilt wurden und damit die räumliche Kongruenz zwischen staatlichen und traditionellen Verwaltungseinheiten aufgehoben wurde. Im Zuge der Dezentralisierung kehrt man nun zu den Nagari als Dorfstrukturen zurück. Diese Reform lehnt sich stark an das Minangkabausche Recht, das Adat Minangkabau, an. Adat ist eine umfassende Kategorie, die das kulturelle, symbolische und rechtliche Universum der Minangkabau andeutet. So kommt es zu einer in dieser Form neuen Begegnung von staatlich-rechtlichen Prinzipien von Demokratie und historisch älteren und nunmehr wieder aktualisierten Minangkabauschen verfassungsrechtlichen Prinzipien. ${ }^{5}$ Dieser „Minangkabausche Weg“" hat auch außerhalb WestSumatras viel Aufmerksamkeit auf sich gezogen, sowohl bei Gebern wie auch bei Vertretern anderer ethnischer oder regionaler Organisationen in Indonesien. Er wird als ein wichtiger Ansatz betrachtet, in dem lokale traditionelle Strukturen und Führer wieder eine größere Rolle in einem Prozess bekommen, der zu mehr Demokratie und einer weitergehenden Einbeziehung der Bürger führen soll. ${ }^{6}$

Meine Frau und ich haben diese Prozesse seit 1999 ziemlich systematisch verfolgt. ${ }^{7}$ Wir kennen das Gebiet recht gut, da wir dort 1974 und 1975 eine 16-monatige Feldforschung gemacht haben. ${ }^{8}$ Seit 1999 sind wir jedes Jahr zwischen 4 und 8 Wochen dort gewesen und

Asia Research Centre, 2001 [Fn. 3].

5 Für detailliertere Beschreibungen unserer Forschungsergebnisse siehe $F$. und K. von BendaBeckmann 2001 [Fn. 1].

„The Governor of West Sumatra in consultation with the local governments is looking at restructuring the administrative system along the lines of traditional governing structures in order to make local government more democratic, improve local government services and increase citizen involvement"; Asia Research Centre [Fn. 3], Appendix F.

Unsere Forschung geschieht in Zusammenarbeit mit der Andalas Universität in Padang. Wir danken Prof. Dr. Aziz Saleh, Alfan Miko MA, Erwin MA, Prof. Dr. Syahmunir, Prof. Dr. Syofyan Thalib, Narullah Dt. Parpatiah nan Tuo SH, MA, Dr. Takdir Rahmadi, and Tasman SH für ihre Hilfe.

Siehe F. von Benda-Beckmann, Property in social continuity: Continuity and change in the maintenance of property relationships through time in Minangkabau, West Sumatra, The Hague, 1979, K. von Benda-Beckmann, The broken stairways to consensus: Village justice and state courts in Minangkabau, Dordrecht, 1984. 
haben den Prozess der Dezentralisierung durch die Zeit verfolgen können; ein Prozess, der übrigens bei weitem noch nicht abgeschlossen ist. Aber wir haben sehen können, wie zunächst im Jahre 2000 die Dezentralisierungsgesetzgebung auf der Ebene der Provinzen konkretisiert wurde, unter anderem durch eine Rahmenverordnung über die neue Dorfverfassung. ${ }^{9}$ Im vorigen und in diesem Jahr (2002) konnten wir verfolgen, wie die Provinzverordnung in Verordnungen der neuen Distrikte übersetzt und weiter konkretisiert wurde. Schließlich haben wir auch in manchen Dörfern die noch weitere Konkretisierung in der Dorfpolitik gesehen. In diesem Prozess werden die politischen und verwaltungstechnischen Befugtheiten und Legitimationen neu gestaltet. Auch kommt es zu einer Veränderung der Autorität über wirtschaftliche Ressourcen, vor allem der Verteilung von Steueraufkommen und der Nutzung der natürlichen Ressourcen. In meinem Beitrag werde ich vor allem auf die politischen, verfassungsrechtlichen Aspekte eingehen, weil es gerade hier zu einem neuen Nebeneinander, Ineinander und Gegeneinander von unterschiedlichen Konzeptionen von politischer Legitimität kommt. Zunächst werde ich die Grundprinzipien Minangkabauschen Verfassungsdenkens rekonstruieren, die in ihrer geschichtlichen Entwicklung vor die koloniale Zeit zurückreichen. Danach will ich die wesentlichsten Entwicklungen bis in die 70er Jahre schildern, um dann etwas ausführlicher auf die jüngste Reform im Zuge der Dezentralisierung einzugehen.

\section{Die wechselhafte Geschichte der Nagari}

\section{Nagari und Adat}

Die Minangkabauschen Nagari (das Wort stammt wie viele Worte der Indonesischen Sprache aus dem Sanskrit und bedeutet eigentlich Staat) werden meist als Dorfrepubliken gedeutet. Nicht jede Siedlung war ein Nagari. ${ }^{10}$ Um den Status eines Nagari zu haben, mussten Siedlungen eine Reihe von Bedingungen erfüllen. Das Gebiet musste ausreichende Ressourcen haben, Reisfelder und Waldgebiete, einen eigenen Markt, einen Versammlungsplatz, ein Rathaus, eine eigene Moschee. Vor allem aber musste es durch zumindest vier Suku bewohnt werden. Suku sind die Minangkabauschen Matriklans, Abstammungsgruppen, deren Kern sich auf wirkliche oder putative gemeinschaftliche Abstammung in der weiblichen Linie gründet. Nach Nagari-Gründung Zugezogene müssen in die lokale Klanstruktur des Nagari aufgenommen werden, um eine, wenn auch nach Adat zweitrangige, Dorfbürgerschaft zu bekommen. Damit haben die meisten Suku eine intern differenzierte Struktur. Die Oberhäupter, Panghulu, der Matriklane, die sich zum Nagari zusam-

Die Lage in den Städten ist einer eigenen Verordnung vorbehalten. Man erwägt, den NagariStrukturen auch innerhalb der Städte eine größere Geltung zu verschaffen. Dies ist allerdings ein besonders umstrittener Punkt in der Regionalpolitik.

10

Das Minangkabausche Adat unterschied vier unterschiedliche Status für Siedlungen. 
mengeschlossen hatten, bildeten zusammen den Dorfrat. Man war aber, wie gesagt, nicht sofort Nagari. Es konnte also gut sein, dass bei Nagari-Gründung die ursprünglichen Matriklans schon intern differenziert und oft in mehrere Klansegmente mit ihren eigenen Panghulu aufgeteilt waren, die einen ebenbürtigen verfassungsrechtlichen Status hatten. Der Dorfrat konnte so zwischen 30 und 60 Panghulu haben, die alle zusammen den Dorfrat konstituierten.

Die ursprüngliche verfassungsrechtliche Logik war, dass es sich um einen freiwilligen Zusammenschluss von im Prinzip unabhängigen und gleichwertigen Großgruppen handelte. Diese behielten auch nach dem Zusammenschluss weitgehend ihre Autonomie. Alle interne Angelegenheiten betreffenden Probleme wurden im Prinzip innerhalb der Gruppe entschieden. Der Nagari und der Dorfrat waren in Angelegenheiten zuständig, die das ganze Dorf betrafen. Beim Dorfrat lagen auch die politischen Kontrollrechte über die Dorf-Güter, vor allem der Teil des Dorf-Territoriums, das nicht permanent land- oder forstwirtschaftlich genutzt wurde (Ulayat). ${ }^{12}$ Der Dorfrat funktionierte auch als Berufungsinstanz in Angelegenheiten, die innerhalb der Gruppe nicht friedlich und einverständlich beigelegt werden konnten. Auf dieser Basis beruhte auch das das Adatdenken beherrschende Prinzip, dass gültige Entscheidungen nur einvernehmlich getroffen werden konnten. Einverständnis, oder mupakat oder sakato, wie die Minangkabau sagen, war das Ergebnis eines oft langwierigen und sehr zeremonialisierten Unterhandlungsprozesses. ${ }^{13}$

Entscheidungsfindung auf der Basis von politischer Gleichheit war also eines der grundlegenden verfassungsrechtlichen Prinzipien. Neben diesem egalitären Prinzip gab es auch ein hierarchisches Prinzip, das an der segmentären Struktur der Klane orientiert war. Je umfangreicher das Klansegment, desto höher die politische Position des Gruppenvertreters, im matrilinearen System also des Mutterbruders, auf der Ebene der größeren Gruppen mit dem Adattitel eines Panghulu. Für die Entscheidung und Behandlung von Problemen und

Dabei gab es in Minangkabau zwei unterschiedliche Systeme. In einem, mehr egalitären, Bodi Caniago genannten, hatten all diese Panghulu die gleichen Rechte. In dem anderen, Koto Piliang genannten, gab es eine unterschiedliche Status. Hier wurde die höchste Gewalt durch die „Panghulu der 4 Suku“ ausgeübt, die Führer der 4 alten Suku oder Klanassoziationen. Unter ihnen bildeten dann die anderen Oberhäupter der Klansegmente bzw. Abstammungsgruppen, den Dorfrat im weiteren Sinne. Für mehr Informationen über die politische Organisation in Minangkabauschen Nagari, siehe T.A.L. Kroesen, Het inlandsch bestuur ter Sumatra's Westkust, Tijdschrift Nederlands Indië, $4^{\mathrm{e}}$ serie (1973), deel 2, 81-109, 208-230; L.G. Willinck, Het Rechtsleven bij de Minangkabausche Maleiërs, Leiden, 1909; G.D. Westenenk, De Minangkabausche Nagari, Mededelingen van het Bureau voor de Bestuurszaken der Buitenbezittingen, bewerkt door het Encyclopedisch Bureau, 3rd print, 1918; F. von Benda-Beckmann, 1979 [Fn. 8].

Das war in manchen Nagari allerdings schon seit langem unter der Hoheit der einzelnen Klane.

Siehe K. von Benda-Beckmann, 1984 [Fn. 8]; F. von Benda-Beckmann, Entscheidungsvorgänge in vorindustriellen Gesellschaften, in: A. Müller (ed.), Gesellschaftliche Entscheidungsvorgänge, Basel, 1977. 
Konflikten galt das Prinzip, dass es zunächst auf der untersten Ebene unter der Leitung des Gruppenoberhaupts gelöst werden musste. Wenn das nicht glückte, ging man eine Stufe weiter bis zum Niveau des ganzen Klan und, wenn es auch da nicht glückte, zum AdatDorfrat. Auf höherem Niveau genommene Entscheidungen gingen dann über dieselbe Hierarchie zu den Betroffenen zurück. Bajanjang naik, batanggo turun, wie die Minangkabau sagen. „Man geht die Stufen hoch, man kehrt über die Treppe zurück“. Auf jeder Entscheidungsebene griff aber wieder das egalitäre Prinzip des gemeinsamen Beratschlagens bis zum Konsensus. Diese Ideen formen das Grundverständnis von Adat-Demokratie und legitimer Herrschaft in Minangkabau.

\section{Die Integration des Islam in die Dorfverfassung}

Diese Regeln wurden durch das Aufkommen des Islam, wahrscheinlich im 16. Jahrhundert, erweitert. Der Islam wurde zunächst als offizielle Religion der Oberschicht angenommen, seine weltlichen Regeln jedoch nicht bzw. nur teilweise. Denn die islamischen Prinzipien von Herrschaft, Familienorganisation und Vererbung widersprachen natürlich den matrilinearen Strukturen des Minangkabauschen Adat. Der Streit zwischen Adat und Islam hat dann auch die Geschichte Minangkabaus nachhaltig beeinflusst. ${ }^{14}$

Zunächst mussten sich islamische Prinzipien dem Adat unterordnen. Religiöse Autorität wurde in das System der Matri-Gruppen eingepasst. Die $S u k u$ hatten ihre eigenen religiösen Ämter, die Malims und Chatibs, die, wie auch das Panghulu-Amt, matrilinear, vom Onkel auf Schwestersöhne, weitergegeben wurden. Zu Beginn des 19. Jahrhunderts kam es dann zu einem blutigen Bürger- bzw. Religionskrieg, in dem eine orthodoxe islamische Bewegung, die Padri, die in ihren Augen ungläubige Gesellschaft reformieren wollten. Sie versuchten, auch die Nagari-Verfassung auf ein theokratisches Modell umzustellen ${ }^{15}$. Auf Ersuchen einiger Adatführer intervenierten die Holländer, die bisher nur einen Handelsposten an der Küste gehabt hatten, in diesen Krieg, schlugen die Padri-Einheiten und verleibten das Minangkabausche Hochland ihrem Kolonialreich ein. In der Zwischenzeit

Über die wechselhaften Beziehungen zwischen Adat und Islam in Minangkabau siehe T. Abdullah, Adat and Islam: An examination of conflict in Minangkabau, Indonesia 2 (1966), 1-24; J. Prins, Adat en Islamietische plichtenleer in Indonesie, Den Haag, 1954; J. Kahn, Constituting the Minangkabau, peasants, culture and modernity in colonial Indonesia, Oxford, 1993; Dobbin, Islamic revivalism in a changing peasant economy: Central Sumatra, 1784-1847, London and Malmö, 1983; F. und K. von Benda-Beckmann, Adat and religion in Minangkabau and Ambon, in: H. Claessen / D. Moyer (eds.), Time past, time present, time future, Dordrecht, 1988; K. Young, Islamic peasants and the state: The 1908 anti-tax rebellion in West Sumatra, New Haven, 1994.

In manchen Nagari, die teils 10 Jahre oder länger unter Padri-Herrschaft gestanden hatten, sieht man noch Erinnerungen an diese Zeit, zum Beispiel territoriale Einheiten innerhalb des Nagari mit den Moscheen als Zentrum, siehe F. von Benda-Beckmann, 1979 [Fn. 11]. 
hatten Adat und Islam zwar Frieden geschlossen, aber es war zu spät, um die politische Unabhängigkeit der Nagari zu gewährleisten. Aber es bildete sich nun ein neues ideologisches Grundprinzip heraus, dass den Zusammenhalt von Adat und Islam zu dem zentralen Merkmal Minangkabauscher Kultur und Identität machte. „Adat beruht auf der Syarak, die Syarak auf Adat". ${ }^{16}$

\section{Die Nagari im Staatsverband}

Zwar hatten die Holländer in ihren ersten Verträgen den Minangkabau zugesichert, sie würden sich nicht in die lokalen Angelegenheiten einmischen, aber, wie das so üblich war, hielt man sich nicht in die Verträge. Die Holländer unterstützten die Adatstruktur bewusst, um damit ein Gegengewicht $\mathrm{zu}$ dem politisch viel virulenteren Islam zu haben. Ihre indirekte Herrschaft nahm die Nagari als Basiseinheiten. Mit 60 gleichberechtigten Panghulu zu unterhandeln, war ihnen jedoch auch zu viel. So wurde ein Dorf-Panghulu quasi als Bürgermeister eingesetzt. ${ }^{17}$ Man hielt sich jedoch zunächst mit Eingriffen in die Dorfstruktur zurück. Vereinzelt kam es zu Veränderungen der territorialen Integrität einiger Nagari. In manchen Fällen wurden große Nagari geteilt oder kleine zu einem größeren zusammengefügt. $^{18} 1914$ bekamen die Nagari dann eine Gemeinde-Verfassung, die auch einen Nagari-Rat installierte, dem allerdings nie alle nach Adat berechtigten Panghulu angehörten.

Syarak ist die Minangkabausche Übersetzung von syari'ah. Die Aussage Adat basandi syarak, syarak basandi adat, deutete auf eine prinzipielle Gleichwertigkeit. Die in jüngerer Zeit bevorzugte Ausdrucksweise adat basandi syarak, syarak basandi kitabullah (,Adat beruht auf Syarak, die Syarak auf dem Koran") will eine leichte Überlegenheit des religiösen Rechts angeben. Im heutigen West Sumatra wird das als ABSSBK abgekürzt.

Auch wurden zur Zeit des Cultuurstelsels, dem Zwangsanbau und Ablieferung von Handelsgewächsen - in Minangkabau war das Kaffee -, eine Reihe von hierfür verantwortlichen Panghulu (rodi) eingesetzt, die oft kein Recht nach Adat auf diese Position hatten. Über die Position der Panghulu siehe auch M. Naim, Penghulu di Minangkabau (Penghulu as Traditional Elite in Minangkabau), Working Paper No. 14, Singapore: Department of Sociology, University of Singapore, 1973; I. Manan, A traditional elite in continuity and change: The chiefs of the matrilineal lineages of the Minangkabau of West Sumatra, Indonesia, PhD thesis, University of Illinois at Urbana-Champaign, Ann Arbor, 1984.

Auch verboten die Holländer die weitere Aufteilung der Panghulu-Gruppen, weil sie damit die bei der Gruppenspaltung regelmäßig auftretenden Konflikte unterbinden wollten. Dies half nur sehr bedingt, denn die Minangkabau handelten weiterhin nach Adat. Die Maßnahme introduzierte jedoch ein Element der Unklarkeit bezüglich der Frage, wer nun ,wirklich“ welche adatrechtliche Position einnahm. Auch wurde zwischenzeitlich (von 1874 bis 1914) eine über den Nagari stehende Verwaltungsebene geschaffen, die Laras unter der Leitung eines Laras-Oberhaupts, siehe W.J. Leyds, Larassen in Minangkabau, Koloniale Studiën 10 (1926), 387-416. 
Im Bereich des Boden- und Ressourcenrechts erkannten die Holländer das Minangkabausche Adat im Rahmen ihres neuen kolonialen Rechtssystems weitgehend an. Im Zuge der Agrarreform in den 1870er Jahren wurden allerdings alle sogenannten „wüsten Gebiete“ (Holl. woeste gronden; Engl. waste lands) zur Staatsdomäne erklärt. Hierunter vielen nach Meinung der meisten Kolonialbeamten und -juristen all die Gebiete, auf die es keine dem holländischen Eigentumsbegriff mehr oder weniger entsprechenden Rechte gab, insbesondere das Ulayat-Land, der nicht systematisch und permanent land- oder forstwirtschaftlich genutzte Teil des Nagari-Territoriums. ${ }^{19}$

Seitdem hatten alle Nagari bis in die 70er Jahre des 20. Jahrhunderts eine mehr oder weniger ausgeprägte dualistische politische Organisation, die auf unterschiedlichen Legitimationen beruhte. ${ }^{20}$ Auf der einen Seite die staatlich geregelte Dorfverwaltung, bei der neben dem Dorfbürgermeister unterschiedliche Formen von Räten und Parlamenten die Dorfbevölkerung repräsentieren sollten. In diesen waren die „Drei Säulen“ vertreten: die Adatältesten (Niniek Mamak), die religiösen Führer (Alim Ulama) und die „Intellektuellen“ (Cerdik Pandai). Daneben gab es einen Dorfrat, der auch als streitschlichtende Instanz auftreten konnte und sollte. In diesem saßen mehrere, aber längst nicht alle Panghulu. Parallel dazu sah man jedoch die Fortsetzung einer, auf jeden Fall ideologisch „reinen“ Adatstruktur, mit dem Adatdorfrat als höchstem Organ, Kerapatan Adat Nagari (KAN) oder Rat der Panghulu genannt, der in Prinzip alle nach Adat berechtigten Panghulu umfasste. ${ }^{21}$ Diese Adatdorfräte waren über lange Perioden durch die Kolonialregierung und nach der Unabhängigkeit durch den Indonesischen Staat nicht offiziell anerkannt, wurden aber in der Verwaltungspraxis geduldet und durchaus geschätzt. ${ }^{22}$

Hierüber gab es allerdings heftige Diskussionen unter den Holländischen Juristen. Vor allem die Vertreter der Adatrechtsschule in Leiden setzten sich für die Anerkennung der Nagari-UlayatRechte ein. Siehe Van Vollenhoven, De Indonesier en zijn grond, Leiden, 1909; J.H.A. Logemann / B. ter Haar, Het beschikkingsrecht der Indonesische rechtsgemeenschappen, offprint from Indisch Tijdschrift voor het Recht 125 (1927), 347-464. Für die Situation in West-Sumatra, siehe F. von Benda-Beckmann, 1979 [Fn. 11]. Indonesian polity, Amsterdam, 1999.

Siehe auch $S$. Thalib, Pemerintahan Nagari di Sumatera Barat Dewasa Ini; suatu tinjauan terhadap SK No. 15/GSB/1968, Padang, 1974. tion in den 1970er Jahren in Nagari Candung Kota Lawas, siehe K. von Benda-Beckmann, 1984 [Fn. 13]; F. von Benda-Beckmann, 1979 [Fn. 11]. 


\section{Die Desa-Reform}

Das blieb im Prinzip so bis 1983, als die Desa-Verwaltung eingeführt wurde. ${ }^{23}$ Hiermit wurden die untersten Verwaltungsstrukturen für ganz Indonesien einheitlich nach dem Modell geregelt, das die Holländer und nach ihnen der indonesische Staat den Desa-Dörfern auf Java nachempfunden hatten. Es bedeutete, dass die Nagari in mehrere Desa aufgeteilt wurden. Die 543 Nagari in West Sumatra Minangkabau wurden so zu 3.516 Desa. Es zeigte sich bald, dass diese Aufteilung zu allzu kleinen Einheiten führte. 1988/9 reduzierte man deshalb die Anzahl der Desa, wonach 1.744 Desa übrig blieben. ${ }^{24}$ Alle Desa hatten einen Bürgermeister und ein Desa-Parlament. Politisch wurde diese Reform durch die staatliche Finanzierungsstruktur erleichtert. Jedes „Dorf“ bekam unabhängig von dem Bevölkerungsumfang eine einheitliche Finanzierung. Da die Minangkabauschen Nagari wesentlich größer als die Dörfer auf Java oder anderswo in Indonesien waren, war es auch aus der Sicht der Lokalpolitiker attraktiv, die Nagari aufzuteilen, um nicht viel weniger staatliche Subventionen zu bekommen. ${ }^{25}$

Der Nagari war also abgeschafft. Aber wiederum auch nicht ganz. Denn auf Provinzebene sah man ein, dass das durch die Desa entstehende Adat-Vakuum irgendwie gefüllt werden musste. Eine Provinzverordnung von 1983 konstituierte und regelte deshalb einen NagariAdat-Rat (Kerapatan Adat Nagari, KAN) und sprach vom Nagari als einer Adatrechtsgemeinschaft, die durch den Nagari-Adat-Rat vertreten werde. Seine Aufgaben waren, die Einheit der Nagari-Bevölkerung zu bewahren, die ideellen und materiellen Werte des Nagari zu verwalten, und Adat-Streitigkeiten zu schlichten. Adat-Streitigkeiten waren im wesentlichen bodenrechtliche Fragen, Erbstreitigkeiten und Konflikte über Adat-Ämter und Panghulu-Titel. Ein Rundbrief des West-Sumatranischen Berufungsgerichts ordnete an, dass derartige Streitigkeiten von den Gerichten nicht akzeptiert werden sollten, wenn nicht zuvor der Adatrat eine Entscheidung getroffen habe. Auch für die dörfliche Entwicklungspolitik wurde in einem ausführlichem Beschluss des Gouverneurs von 1990 auf die Nagari als die relevanten Einheiten zurückgegriffen. Schließlich wurden dem Nagari-Adat-Rat auch weitreichende Befugnisse über die Nagari-Ressourcen zugestanden. So hatte man eine interessante und beinahe paradoxe Situation. Der Nagari als offizielle Verwaltungseinheit war abgeschafft, als ,informelle“ soziale Einheit jedoch anerkannt und sehr detailliert durch den Staat geregelt. Was als Nagari-Adat-Rat anerkannt und konstituiert wurde, war jedoch wiederum ein ganz anderes Gebilde als der seit langem nicht mehr anerkannte „reine“ Adatdorfrat, so wie wir ihn in den 1970er Jahren erlebt hatten. Vorsitzende des neuen Rates

Die Basis hierfür war in zwei Gesetzen von 1974 und 1979 gelegt worden.

24

25

Der Nagari, in dem wir 1974-75 geforscht hatten, wurde erst in 13, dann in 6 Desa aufgeteilt.

Siehe auch M. Galizia, Village institutions after the law No. 5/1979, Archipel 51 (1996), 135-160,

T. Kato, Different fields, similar locusts, Indonesia 47 (1989), 89-114. 
wurden meist durch politisch gefällige und der Staatspartei GOLKAR ergebene Adatführer, oft Beamte, Lehrer etc.

\section{Die Dezentralisierungsreform von 1999 und später: Zurück zum Nagari}

\section{Allgemeine Entwicklung}

Die Desa-Reform wirkte sich nicht positiv auf das soziale und wirtschaftliche Leben aus. Die soziale Einheit des Nagari wurde geschwächt. Die neuen administrativen Grenzen liefen quer durch die sozialen und wirtschaftlichen Strukturen des Nagari. Die traditionell wichtigsten Gruppenmitgliedschaften und Familienbeziehungen basierten auf matrilinearen, über die weibliche Linie bestimmten Abstammungsbeziehungen. Kinder gehörten demnach nicht zu der Gruppe ihres Vaters, sondern zu der Mutter. Nicht der Vater oder Großvater war die Autoritätsperson, sondern der Onkel oder Großonkel mütterlicherseits. Zwar sind im Laufe der letzten 100 Jahre die sozialen Beziehungen innerhalb der Kleinfamilie sehr viel intensiver geworden, aber in Adat-Angelegenheiten, bei der Nachfolge in Führungspositionen und der Kontrolle und Nutzung des matrilinearen Erbvermögens, vor allem der Nassreisfelder, blieb die Mitgliedschaft in der matrilinearen Gruppe von größerer Bedeutung. Auch war es, und ist es überwiegend auch heute noch im Dorf die (Adat- und statistische) Regel, dass ein Mann nach seiner Hochzeit in das Haus seiner Frau zieht, das auf dem Familienland seiner Frau steht. Dies war eines der Probleme, das die Autorität der DesaBürgermeister tangierte. Denn sie wohnten oft in der Desa ihrer Frau, wo sie als Eingeheiratete kaum Autorität nach Adat beanspruchen konnten. Ihre Autorität, ihr Mitspracherecht war dort, in der Desa, wo ihre eigene Abstammungsgruppe ihre politische Residenz hatte und wo sich auch meist das Erbvermögen ihrer eigenen Abstammungsgruppe befand. Die bürokratische Desa-Verwaltung hatte also meist überhaupt keine Parallele in entsprechenden Befugnissen nach Adat, so wie das auf dem Niveau des Nagari noch weitgehend der Fall gewesen war, wenn, was durchaus üblich war, man innerhalb des Dorfes geheiratet hatte. Auch hatten die Desa-Bürgermeister keine Autorität über die Ressourcen des ehemaligen Nagari, die dem Adatdorfrat übertragen worden war. Aber auch diese Dorfräte waren kein Erfolg. Es häuften sich kritische Stimmen, die darauf hinwiesen, dass der Rat und die Panghulu zu leichtfertig, zu sehr im eigenen Vorteil und ohne Konsultationen mit ihren Gruppenmitgliedern über die Nagari-Ressourcen verfügten und oft zwielichtige Geschäfte mit Investoren oder Regierungsinstanzen machten. Auch als streitschlichtendes Forum schienen diese Dorfräte nicht besonders gut zu funktionieren.

Die größere politische Freiheit nach dem Ende des Suharto-Regimes und die sich ankündigende Dezentralisierungspolitik führten dazu, dass die Diskussionen über die Beziehungen zwischen und Vor- und Nachteile von Nagari und Desa wieder aufflammten. Schon vor Ausfertigung der nationalen Dezentralisierungsgesetze hatte sich die Provinz-Regierung 
von West-Sumatra den Slogan „Zurück zum Nagari“ zu eigen gemacht, vor allem um gegenüber dem Javanisch beherrschten Zentrum die eigene kulturelle und politische Eigenständigkeit zu betonen. ${ }^{26}$ Was das nun konkret heißen sollte, war jedoch undeutlich, und in der Folgezeit entwickelten sich heftige Diskussionen in unterschiedlichen Arenen, in der Verwaltung auf unterschiedlichen Verwaltungsebenen, in der regionalen Presse, der Universität und auch auf Dorfebene. Zu welchem Nagari würde man zurückkehren? Zu vorkolonialen Formen? Zu kolonialen Formen? Zu Konstruktionen, in denen politische Partien das politische Leben im Dorf dominiert hatten, zur Dorfverfassung, so wie sie vor der Desa-Reform existiert hatte? Wie würde sich das auf die Rolle der Panghulu und des Adatdorfrats auswirken?

Es ist eine spannende Geschichte, die hier leider nicht in der Ausführlichkeit, die sie verdient, nacherzählt werden kann. ${ }^{27}$ Im Jahre 2000 wurde der, inzwischen 20. Entwurf der Provinzverordnung für die Dorfverfassung durch das Provinzparlament angenommen. Dies war ein Rahmengesetz, das die Kernstruktur der neuen Dorfregierung regelte. Diese wird aus einem Bürgermeister und einem Dorfparlament bestehen. Daneben soll es einen „Konsultativen Adat- und Syarak-Rat“ und eine „Dorf-Adat-Institution“ geben. Die weitere Konkretisierung dieser Prinzipien wurde den Distrikten überlassen.

Bei der inhaltlichen Ausarbeitung dieser Neugestaltung und dem an den Tag gelegten Tempo zeigten sich große regionale Unterschiede. Manche Distrikte haben zügig mit der Reorganisation und dem Aufbau der neuen Dorfverwaltungen begonnen. Andere sind eher passiv und sehen in der neuen Verwaltungsstruktur eine Bedrohung ihrer erworbenen politischen und wirtschaftlichen Privilegien. ${ }^{28}$ Die zwischen 2000 und April 2002 erlassenen Distriktsverordnungen weisen auch deutliche Unterschiede in der Gewichtung von Adat gegenüber Islam auf. So wurde in einem Distrikt (Tanah Datar) auf die Errichtung des Konsultativen Adat- und Syarak-Rats verzichtet, und der Adatdorfrat mit weitergehenden Befugnissen ausgestattet. In zwei anderen Distrikten (Agam, 50 Koto) dagegen wurden neben dem Adatdorfrat auch ein eigener „Rat der religiösen Führer“ errichtet.

Auch auf Dorfebene sieht man unterschiedliche Entwicklungen. In manchen Dörfern wollen sich nicht alle Anfang der 80er Jahre aufgeteilten Desa wieder zu einem Nagari zusammenschließen. Die meisten neuen Dörfer wissen noch nicht, woran sie finanziell sein

Schon 1998 teilte der Governeur der Provinz dem Innenministerium mit, dass man gedenke, im Rahmen der zukünftigen Möglichkeiten zur Nagari-Struktur zurück zu kehren, und damit auch zu einer besseren Anpassung der staatlicher Dorfverwaltung an Minangkabausche sozial-kulturelle Werte zu kommen.

Für mehr Details siehe F. und K. von Benda-Beckmann, 2001 [Fn. 1]. 
werden, da weder die benötigten Finanzregelungen noch die Regelungen der Dorfverwaltung schon existieren. Es gibt Dörfer, die sich schon vor der Gesetzesänderung weitgehend Gedanken darüber machen, wie sie ihre Verwaltungs- und Finanzstruktur gestalten wollten. In anderen Dörfern wird sehr abwartend auf die neuen Regelungen reagiert. Im Frühjahr 2002 waren in zwei Distrikten in fast allen Dörfern die Bürgermeister und Dorfparlamente gewählt. Manche Distriktschefs waren bereits dazu übergegangen, den Nagari nach dem neuen finanziellen Modell Gelder zuzuweisen, andere noch nicht.

Ich werde mich im folgenden vor allem auf drei Fragenkomplexe beschränken. Die Art der politischen Repräsentation in den offiziellen Organen der Dorfverfassung; die Rolle des Adatdorfrats; und die Probleme bei der Wiedervereinigung mancher Nagari, weil vor allem die darüber geführten Diskussionen und entsprechenden Praktiken viel über das unterschiedliche Demokratieverständnis in Minangkabau aussagen.

\section{Das Dorfparlament}

Die Provinzverordnung spricht von einem Dorfparlament, einem Rat von Repräsentanten der Dorfbevölkerung, ${ }^{29}$ die in freien, allgemeinen und geheimen Wahlen gewählt werden sollen. Diese Regel wurde jedoch in den Distriktsverordnungen und Dorfverfassungen in zweierlei Hinsicht modifiziert.

Einmal wird eine Differenzierung der allgemeinen Kategorie von Dorfbürgern in eine Vielzahl von sozialen Kategorien von zu repräsentierenden Gruppierungen vorgenommen. Bei diesen Kategorien sind die drei klassischen Säulen: Adatälteste, religiöse Autoritäten und Intellektuelle allgegenwärtig und dominant. Fast immer gibt es daneben die Kategorien der „Adat-Frauen“ und der „Jungen“. Manche Distrikts- und Dorfverordnungen operieren zusätzlich mit den Kategorien „lokale Führer“, „Professionals“, „Bauerngruppen“ und „Migranten“, d.h. aus dem Dorf stammende, aber außerhalb des Dorfes lebende Dorfbürger. ${ }^{30}$ Zum anderen kommt es zu einer Modifizierung des Wahlmechanismus, der von direkten und geheimen Wahlen bis zum nach Adat klassischen Selektionsverfahren durch gemeinschaftliche Beratschlagung bis zum Konsens (musyawarah untuk mupakat) reicht. Ein allgemeines Element ist, dass jede ehemalige Desa einen Repräsentanten in das Dorfparlament schicken kann. Die anderen Mitglieder werden meist nach sozialen Kategorien

Der Art des Parlaments und die maximale Anzahl seiner Mitglieder sind in der Provinzverordnung geregelt. Diese überlässt die weitere Ausgestaltung jedoch den Distriktsverordnungen. Einige derselben legen eindeutige Kriterien fest, z.B. x Vertreter pro 1.000 Einwohner; andere überlassen dies den Dorfverordnungen selbst.

Dabei sind die Einpassung in diese Kategorien flexibel. Ein junge Lehrerin kann zum Beispiel „Frau“ oder „Intellektuelle“ sein. 
ausgewählt, so wie die Provinzverordnung das eigentlich nur für den Konsultativen Adatund Syarak-Rat vorsieht. ${ }^{31}$ Der Wahlmechanismus ist dabei meist ein Prozess der konsensualen Entscheidungsfindung nach Adat. Das heißt, dass Adatrepräsentanten unter und durch die Adatältesten ausgesucht werden, die religiösen Führer ebenfalls aus dem eigenen Kreis, usw.

Diese Auswahlverfahren werden auf allen Politik-Ebenen ausführlich diskutiert. Manche klagen über ein undemokratisches Verfahren, das durch die alten und neuen Eliten dominiert würde. Die Verteidiger des Adat protestieren vehement, wenn gesagt wird, diese Verfahren seien nicht demokratisch. Die Minangkabausche Demokratie sei besser als die westlichen Mehrheits-Demokratien. Es ist nicht nur eine altmodische traditionalistische Adat-Elite, die die Minangkabausche Demokratie preist. Auch in öffentlichen Diskursen „moderner“ Politiker wird die Minangkabausche Demokratie gepriesen. Der Rektor der Universität von Padang, Professor der Medizin, erklärte, dass die Bedingungen für und Auswahlverfahren von den Mitgliedern des Dorfparlaments nach Adat geschehen müsse. Und der Distriktschef von Solok, einer der dynamischsten Distriktschef in West Sumatra, und in ganz Indonesien als einer der fortschrittlichsten Umsetzer der Dezentralisierungspolitik bekannt, meinte, dass die Demokratie nach Minangkabauschem Vorbild höher als die westliche Demokratie bewertet werden müsse. Die Nagari seien ja Republiken gewesen, und das habe der Westen erst von Minangkabau gelernt.

In einem Punkt sind sich jedoch, zur Zeit, alle einig. Parteiendemokratie soll es auf Dorfebene nicht geben. Parteiendemokratie wird mit dem alten Regime assoziiert und als undemokratisch empfunden. ${ }^{32}$ Eine ausbalancierte Vertretung könne eben am besten über die Adat-Mechanismen, soziale Kategorien und Konsensusfindung erreicht werden. Die heutige Regelung nimmt auch die drei klassischen Adat-Kategorien als Anknüpfungspunkt. Ihre Erweiterung ähnelt jedoch auch sehr dem Funktionalen-Gruppen-Modell, auf das sich während des Suharto-Regimes die GOLKAR Regierungspartei gründete. ${ }^{33}$ Diese Parallele

Der, politisch wohl nicht so wichtige Adat- und Syarak-Rat, repliziert die klassische Dreiteilung, die drei Säulen des Minangkabauschen Adat: Die Adatältesten, die religiösen Autoritäten und die Intellektuellen. Man spricht dann auch von dem „Rat der Drei Säulen“, vom Majelis Tungku Tigo Sajarangan, eine Nomenklatur, die auch in manche Distriktverordnungen übernommen ist. Die Provinz-Rahmenverordnung erwähnt weiter auch noch ,die Frauen“ und „,andere Komponenten der Gesellschaft“. Auf Dorfebene gibt es eine reichere Variationsbreite. Manche belassen es bei der klassischen Dreiteilung. Andere sind differenzierter in ihrer Kategorieformung; siehe $F$. and K. von Benda-Beckmann, Recreating the nagari, 2001 [Fn. 1].

Auf allen höheren Ebenen gibt es sie natürlich, noch bzw. wieder in einem sehr schlechten Ruf wegen der alles durchdringenden money politics und der Tatsache, dass Parlamentarier offensichtlich nichts besseres zu tun haben, um sich selbst erstaunliche Vergütungen zuzubilligen und sich mehr den Parteien als den Wählern verpflichtet zu zeigen. 
wird jedoch nicht gesehen. Es geht um Adat. Und diese Verfahren Adat zu nennen, schließt auch gut an die dominante entwicklungspolitische Ideologie an, wonach die Partizipation lokaler Gemeinschaften, gestützt auf lokales Recht, einen hohen Stellenwert hat. Adat ist „Entwicklung von unten“. Viele Nicht-Regierungsorganisationen unterstützen deshalb auch diese Entwicklung; auch die GTZ, die die Indonesische Regierung in der Dezentralisierungspolitik berät, und deren Mitarbeiter in drei ausgewählten Dörfern Workshops zur Erstellung von Dorfverfassungen organisierten.

\section{Der Adatdorfrat (KAN)}

In der Provinzverordnung hatte man sich nach vielen kontroversen Diskussionen nicht für den Namen Adat-Dorf-Rat (KAN) entschieden, sondern für „Adat-Dorf-Institution“ (Lembaga Adat Nagari, LAN). Der dafür angeführte Grund war, dass man sich vom KAN distanzieren wollte. Nicht, weil der KAN eine Adat-Institution war, sondern weil er, in den Augen dieser Betrachter, erst 1983 durch das Suharto-Regime eingeführt worden war und mit dem undemokratischen Regime und den regimehörigen Adatfunktionären assoziiert wurde. Wo also hier die Bindung und politische Verschmutzung des ,alten“ KAN und der neue Charakter der Adatdorfinstitution hervorgehoben wird, betonen andere wieder die historische Tradition. Der KAN sei seit jeher ein zentrales Element im Nagari und Adat gewesen. Es sei Unsinn zu behaupten, es gebe ihn erst seit der Schaffung oder Anerkennung durch den Gouverneur im Jahre 1983. Diese Regelung habe lediglich die Legitimität und Funktion des KAN verstärkt. Wollte man nun den KAN abschaffen? Was wären denn die LAN, wenn nicht die KAN?

Die provinzielle Rahmenverordnung ließ den Spielraum, andere institutionelle Namen zu wählen. Die meisten Distriktsverordnungen wählten dann auch KAN; nur zwei Distrikte entschieden sich für LAN. Auf Dorfniveau übernahm man im Prinzip die Nomenklatur der Distriktsverordnungen.

Die KAN/LAN werden in allen Distriktsverordnungen qua Zusammensetzung und Funktionen geregelt. Die Funktionen sind meist auf die Behandlung von Adatstreitigkeiten beschränkt und auf die Aufrechterhaltung und Stärkung von Adat. Aber obwohl deutlich durch den Staat geregelt, ist der KAN damit nicht zu einem Teil der offiziellen Dorfverwaltung geworden. Manchen kommt das wie eine bewusste Diskriminierung vor, eine Ausschließung des KAN, und damit des Adat, aus der offiziellen Dorfpolitik; AusschlieBung auch von der formellen Teilnahme an Macht. Andere betonen demgegenüber, dass diese Nicht-Anerkennung gerade gut sei, weil sie dem KAN erlaube, unabhängig von offiziellen politischen Zwängen zur Dorfpolitik und zu Adatfragen Stellung zu nehmen. 
Hinzu kommt, dass die Regelung der KAN von 1983 durch die neue Provinzverordnung abgeschafft wurde. Das heißt, dass die dort niedergelegten Befugnisse des KAN über die Dorf-Ressourcen sich im förmlichen Recht nicht mehr finden. Und hierüber geht nun, je weiter der Gestaltungsprozess fortschreitet, der Streit. Wem gehören, und wer kontrolliert die Reichtümer des Nagari, die Aset Nagari, das Einkommen aus Verkauf oder Verpachtung von Nagari-Ressourcen, Holz, Steine, Sand, Wasser? Dem Nagari, nach Adat durch den KAN vertreten, oder dem Nagari, vertreten durch die neue Dorfregierung? Vor zwei und drei Jahren waren das noch ziemlich hypothetische Fragen. Aber bei unserem letzten Besuch im Frühjahr 2002, als manche Distrikte ihre ersten finanziellen Zuweisungen an die Dörfer gemacht hatten, die Nagari ihre ersten Budgetpläne machten, in manchen Distrikten die Kontrolle über die Märkte und Markteinkommen an die Nagari zurückgegeben bzw. delegiert wurden, waren sie zu Fragen von großer wirtschaftlicher Bedeutung geworden. Vor allem ist auch der alte Streit, wer rechtmäßiger Eigentümer der Ulayat-Ressourcen ist und ob Staatsland nicht wieder in Ulayat umgewandelt werden müsse, in neuer Heftigkeit losgebrochen. In Gerichtsprozessen, Unterhandlungen und manchmal gewalttätigen Formen der Selbsthilfe verlangen Nagari bzw. Bewohner von Nagari ihre vom Staat ,gestohlenen“ und häufig an private Unternehmer übertragenen Ressourcen zurück, teils mit Erfolg. ${ }^{34}$ Das alte Adatrecht wird hier gegenüber dem staatlichen Recht mobilisiert. Diese ersten kleinen Erfolge in Ulayat-Angelegenheiten macht die Frage, wer diese Rechte im Nagari kontrolliert, wieder zu einem wichtigen Punkt in der Dorfpolitik. Soweit wir das während unseres letzten Besuchs sehen konnten, gibt es sehr unterschiedliche Praktiken, die von einem guten Einverständnis zwischen den neu gewählten Bürgermeistern und Dorfparlamenten und den Adatführern und den KAN bis hin zu offenen Konflikten reichen.

\section{4. $\quad$ Probleme bei der Wiedervereinigung der Desa zu Nagari}

Spannungen zwischen modernen Gleichheitsidealen und historischen Minangkabauschen sozialen und rechtlichen Unterschieden zeigten sich auch bei der Frage des Zusammenschlusses der Desa. Vorsichtshalber hatte die Provinzverordnung dekretiert, man müsse zu der Situation von vor 1983 zurückkehren. Eventuelle Aufteilungen oder Zusammenlegungen von Nagari sollten unter bestimmten Bedingungen möglich sein, aber einem späteren Zeitpunkt vorbehalten bleiben. Es zeigt sich bald, dass nicht überall alle Desa wieder zu ihrem ursprünglichen Nagari zurück wollten. Es gab drei Arten von Problemen, zwei davon politisch eher unschuldig. Das waren erstens Fälle, in denen die wiedervereinigten Nagari viel zu groß geworden wären - teils mit einer Bevölkerung von 60.000 Einwohnern. Zweitens wollten manche Desa, die einmal eigenständige Nagari gewesen, in der

Siehe F. und K. von Benda-Beckmann, 2001, [Fn. 1]; R. Biezeveld, Nagari, Negara dan Tanah Komunal di Sumatra Barat, in: F. and K. von Benda-Beckmann / J. Koning (eds.), Sumber Daya Alam dan Jaminan Sosial, Yogyakarta, 2001, 135-164. 
kolonialen Zeit oder danach jedoch zusammengefügt worden waren, ihre ursprüngliche Identität zurückerlangen. Drittens, und am interessantesten und konfliktreichsten sind die Fälle, in denen Desa überwiegend durch Personen oder Gruppen bewohnt werden, die im Adatsystem Zugezogene und/oder die Nachkommen ehemaliger Sklaven waren. ${ }^{35}$ Im Minangakabauschen Adat hatten diese einen niedrigeren Status. Ihre wirtschaftlichen Rechte waren beschränkt und an die Patron-Klient-Beziehungen zu den Abstammungsgruppen gebunden, in die sie aufgenommen waren und von denen sie Land für Landwirtschaft im Teilanbau bekommen hatten. Da sie keine ursprüngliche Abstammungsgruppe und innerhalb ihres Klans untergeordnet waren, konnten sie auch keinen der im Adatdorfrat vertretenen Panghulu stellen, waren also auch politisch marginalisiert. Mit der DesaReform hatten diese Siedlungen einen höheren Grad an politischer und wirtschaftlicher Unabhängigkeit erhalten, weitgehend frei von Adatbeschränkungen, da die alten Patrone jetzt ja in einem anderen Dorf wohnten. In den Desa stellten sie auch meist den Desa-Bürgermeister. Sie fürchteten nun, dass sie wieder unter die Fuchtel des Hauptdorfs und seines Adatrats kommen würden und dass die wirtschaftlichen Zügel wieder stärker angezogen würden. Sie protestierten gegen die Wiedervereinigung, und probierten, in einigen wenigen Fällen erfolgreich, diese zu sabotieren. Die alteingesessenen Abstammungsgruppen des Haupt-Dorfes waren aus denselben Gründen dagegen, dass diese Desa eigenständige Nagari werden sollten oder wollten. Sie befürchteten, recht realistisch, dass ihnen die Herrschaft und Kontrolle der Gruppen bzw. Nagari-Ressourcen weiter aus den Händen genommen würde.

So sieht man, wie auch hier alte Adatprinzipien, die unterschiedliche wirtschaftliche und politische Status innerhalb des Dorfes konstituierten und die im Laufe der letzten 20 Jahre unter anderem durch die Desa-Reform abgeschwächt worden waren, wieder aktualisiert und zum Gegenstand heftiger, manchmal gewaltsamer Auseinandersetzungen werden. Die Distriktverwaltungen gehen unterschiedlich mit diesem Problem um. Manche (Distrikt Solok) unterstützen die Wiedervereinigung auch der unwilligen Desa vorbehaltlos, unter anderem weil diese Desa nicht die Kriterien erfüllen, die nach Adat für einen Minangkabauschen Nagari gelten. Andere (Distrikt Sawahlunto Sijunjung) ergreifen dahingegen für die unwilligen Desa Partei und ermöglichen ihnen die Selbstständigkeit.

Diese Betonung von Bürgerschaft nach Adat gegenüber dem politischen Gleichheitsideal der Staatsbürgerschaft führt leicht zu Diskriminierung und Ausschließung. Diese Tendenzen lassen sich auch auf anderen Niveaus beobachten. Sie kommt in einer stärkeren Betonung der Ethnizität und in dem Gefühl zum Ausdruck, dass West Sumatra „eigentlich“ Minangkabau ist, und dass Minangkabausche Ressourcen eigentlich für die Minangkabau 
sind. Fremde sind zwar wohlgelitten, haben sich jedoch wie früher dem Adat anpassen der Minangkabauschen Leitkultur sozusagen. Die Provinz West-Sumatra ist zwar in ethnischer Hinsicht ziemlich homogen, aber es gibt mehrere Siedlungen von Transmigranten aus Java und Nord-Sumatra, die unabhängige Desa waren, nun aber auch dem Nagari-Modell entsprechen sollen und dabei nicht nur an den verwaltungsrechtlichen Bedingungen des Staates, sondern auch an denen des Adat gemessen werden. Innerhalb der ethnischen Kategorie Minangkabau sieht man weiter das Aufkommen eines Lokalismus, der nicht mehr Ethnizität zum Anknüpfungspunkt von Unterschieden macht, sondern die Zugehörigkeit zum Distrikt oder Dorf. Diese Tendenzen kann man vor allem bei der durch die Dezentralisierung erforderlich gewordenen Reduktion und Umverteilung des staatlichen Verwaltungsapparats beobachten. Zwar können Beamte nicht entlassen werden. Die Gehaltsstruktur erlaubt es jedoch, dass viele überflüssig gewordene Beamte mit nur ihrem Basisgehalt, das einen Bruchteil des gesamten Gehalts ausmacht, zu Hause sitzen. Zentrum, Provinz, Distrikt und Dorf versuchen, sich gegenseitig überflüssige Beamte zuzuschieben. Bei der Auswahl derjenigen, die im Amt bleiben dürfen bzw. durch die neuen Distriktsbehörden übernommen werden, zeigt sich diese Neigung zum Lokalismus sehr deutlich.

\section{Veränderungen im Rechtspluralismus}

In diesen Prozessen hat sich das relative Gewicht von bürokratischen und staatlichen demokratischen Rechtsformen einerseits und von adatrechtlichen politischen und konstitutionellen Formen andererseits sicher etwas zu Gunsten des Adat geändert. Am Auffallendsten ist dies im symbolischen und ideologischen Bereich. Adat ist wieder ,in“. Für die regionalen Politiker dient die Berufung auf Adat dazu, gegenüber der Zentralregierung die Eigenständigkeit Minangkabaus zu betonen. Die Provinzverordnung und die Distriktsverordnungen sind voller Adat-Rhetorik und berufen sich regelmäßig auf Adat-Maximen und Regeln - auch wenn (und vielleicht gerade weil) das nicht unbedingt in einer konkreten rechtliche Stärkung des Adat zum Ausdruck kommt. Adat wird, und ist zur Zeit noch, die Zauberformel, mit der alle sozialen Übel aus der Welt geschaffen werden sollen: Die Einheit und der Zusammenhalt innerhalb der Nagari soll verstärkt werden, die Jungen werden die Älteren wieder respektieren, die Adatführer die Nagari-Ressourcen nicht mehr an externe Investoren verscherbeln. Diese Rhetorik und die häufig damit verbundene romantische Verklärung der Adat-Vergangenheit hört man vor allem von Mitgliedern der älteren Generation, und sie wird vor allem durch die alten und neuen Eliten getragen. Die jüngere Generation, die nicht mehr erlebt hat, wie das Leben im Nagari war, ist sehr viel skeptischer und steht der Adat zugeschriebenen Bedeutung eher ablehnend gegenüber. Aber Adat hat wieder neues Prestige, und man sieht überall, wie lange Zeit unbesetzte PanghuluÄmter wieder besetzt werden. Allerdings geht es dabei, wie schon in den letzten zwei Jahrzehnten, häufig um Personen, die nicht mehr im Dorf aufgewachsen sind, sondern außerhalb ihrer Nagari wohnen und dort als Beamte, Dozenten oder Händler Karriere gemacht 
haben. Ihre Kenntnis des Adat ist oft beschränkt, vor allem des Nagari-spezifischen Adat, der Nagari-Geschichte, die politische und wirtschaftliche Konstitution des Nagari, die Teilung der Klane und Abstammungsgruppen, ihres Vermögens und ihrer Adat-Funktionen. Und dies ist die Kenntnis, die für eine „traditionsgetreue“ Revitalisierung von Adatstrukturen erforderlich wäre. Diese Kenntnis wird immer weniger im traditionellen Lehrer-Schüler-Verhältnis im Dorf übertragen und kann auch nicht durch die zunehmenden Publikationen über das Minangkabausche Adat - im Sinne der allgemeinen Regeln und Prinzipen - ersetzt werden. Das führt dazu, dass es bei der Revitalisierung von Adat nicht nur um eine „Aktualisierung“ ${ }^{36}$ historisch älterer Adatformen geht, sondern verstärkt um eine „Erfindung“, in der vermeintliche historische Formen selektiv und bewusst der gegenwärtigen politischen Situation und den Zielen der betreffenden Akteure angepasst werden von Bauern, Adatältesten, Universitätsdozenten und Verwaltungsbeamten. Dabei werden alte, teils ,reine“ Adatformen rekonstruiert. Im Bereich der Dorforganisation werden diese dann als Elemente in die staatlichen Regelungen eingegliedert. Im Bereich der Rechte auf natürliche Ressourcen geht diese Rekonstruktion und Revitalisierung von Rechten auf Dorfgrund und Ressourcen am weitesten. ${ }^{37}$ Es ist zur Zeit noch nicht abzusehen, welchen Einfluss dies auf den bisherigen Dualismus zwischen staatlich geregeltem Adat und nichtanerkannten Adat haben wird. ${ }^{38}$

\section{Schluss}

Der Prozess der Dezentralisierung ist noch im vollen Fluss, und es ist sicher verfrüht, um zu einer ausgewogenen Bewertung der Dezentralisierung und Reorganisation der Dorfverfassung zu kommen. Doch lassen sich einige vorläufige Schlussfolgerungen ziehen.

C. Giordano, The past in the present: actualised history in the social construction of reality, in: $D$. Kalb / H. Marks / H. Tak (eds.), Historical anthropology: the unwaged debate, Focaal, vol. 25/27 (1996), 97-107.

37 Für ähnliche Prozesse in anderen Regionen Indonesiens, siehe G. Acciaioli, The re-emergence of customary claims to land among the To Lindu of Central Sulawesi: The revitalisation of Adat in the era of Reformasi in Indonesia, Paper presented at the Annual Conference of the Australian Anthropological Society, 19-23 September 2000, The University of Western Australia, Perth, 2000; T.M. Li, Masyarakat adat, difference, and the limits of recognition in Indonesia's forest zone, Modern Asian Studies 35 (2001) 3, 645-676. Einen guten Überblick über die bodenrechtlichen Probleme in Indonesien gibt T. Lindsey (ed.), Indonesia: Law and society, Leichhardt, 1999.

Diese Art, mit Geschichte und Gewohnheitsrecht umzugehen, ist auch in Minangkabau nicht neu. Siehe dazu K. von Benda-Beckmann, 1984 [Fn. 13]; F. von Benda-Beckmann, 1979 [Fn. 11], Kahn, 1993 [Fn. 14]. 
Auf lokalem Niveau ist die Rückkehr zum Nagari und damit zu einer sehr viel größeren Verwaltungseinheit auf jeden Fall eine wichtige und wahrscheinlich bleibende Veränderung. Sie schafft allerdings in den Fällen, in denen sich Desa der Wiedervereinigung widersetzen, ihre eigenen Probleme. Die Autonomie der Nagari ist verglichen mit den Desa sicherlich größer geworden; die Autonomie vieler Desa gegenüber der neuen NagariRegierung hat jedoch oft abgenommen. Inwieweit die normale Bevölkerung von der neuen Dorfverfassung profitieren wird, ist noch nicht deutlich. Als der Distriktschef von Solok den Bürgermeistern und Dorfparlamentariern im Geiste der Dorfautonomie und Demokratie eine Liste von 105 Befugnissen übergab, meinten die Dorfpolitiker, dass es sich ja eigentlich um eine Liste von Pflichten und Aufgaben handele. Die Distriktchefs entwickeln einen unbändigen Regelungsdrang. Der Erlass von Verordnungen ist, wie man jetzt sagt, zu ihrem Hobby geworden. Trotz aller rhetorischen Bekenntnisse zum development from below sind die meisten Prozesse und Initiativen ziemlich top down. Das schafft Frustrationen und Resignation und gibt nach einer ersten Euphorie wieder Raum für den gewöhnten politischen Zynismus. Andererseits sieht man auch tatsächliche lokale Eigeninitiativen, Distriktschefs und Dorfpolitiker, die neue Ideen und Initiativen entwickeln, den neuen politischen und wirtschaftlichen Freiraum aktiv nützend und sich nicht um die überkommenen hierarchischen politischen Beziehungen kümmern.

Auf höherem Niveau haben sich die politischen und wirtschaftlichen Machtbeziehungen zwischen dem Zentrum und den Regionen sicher zum Vorteil der Regionen geändert. Man fürchtet allerdings den backlash des Zentrums. Befugnisse von großem wirtschaftlichen Interesse, wie die Kontrolle über natürliche Ressourcen, die weitgehend auf die regionalen autonomen Verwaltungen übertragen werde sollten, werden nicht wirklich übertragen. Es gibt Gegenbewegungen im Zentrum und in der Lobby der Provinzgouverneure, die die Entwicklung am liebsten rückgängig machen wollen. Auch manchen Außenstehenden schlägt der Schreck ums Herz. Die Wahl der Distrikte als wichtigste autonome Einheit ist problematisch: das politisch-räumliche Niveau zu klein, die politische Koordination zu schwierig, wenn jeder Distrikt seinen eigenen egoistischen Gang geht und die wirtschaftliche Situation der an Menschen und natürlichen Ressourcen armen Distrikte schwerer wird. In der Zentralregierung gibt es viele Stimmen, die sagen, die Dezentralisierung sei viel zu schnell und zu schlecht vorbereitet in Angriff genommen und viel zu weit gegangen. In Jakarta arbeitet man zur Zeit an einer, allerdings heftig umstrittenen, Revision der Dezentralisierungsgesetze. In einem Leitartikel der Jakarta Post vom 21. Dezember 2000 wurde die Dezentralisierung als „das Bauen eines Schiffes während der Segeltour“ angedeutet. Daran ist wahrscheinlich etwas, bloß wird es schwierig sein, das Schiff der Dezentralisierung zwischendurch aufs Trockendock zu hieven. Die einmal in Gang gesetzten regionalen und lokalen Prozesse können dadurch sicher beeinflusst, wahrscheinlich nicht aber rückgängig gemacht werden. Die nächsten Jahre werden es zeigen. 